\title{
Infrared spectra of C-type variables with ISO
}

J. Hron, R. Loidl

Institut für Astronomie, Univ. Vienna, Türkenschanzstraße 7, A-1180 Wien, Austria

S. Höfner ${ }^{1}$, U.G. Jørgensen

Niels Bohr Institute, Astronomical Observatory, Juliane Maries Vey 30, DK-2100 Copenhagen, Denmark

B. Aringer, F. Kerschbaum, W. Windsteig

Institut für Astronomie, Univ. Vienna, Türkenschanzstraße 7, A-1180 Wien, Austria

\begin{abstract}
We present ISO-SWS spectra for several representative AGB carbon stars obtained at several phases of the pulsational cycle. For stars with significant mass loss we correct the spectra for the dust emission by using DUSTY models. We then compare these corrected data to synthetic spectra based on hydrostatic and dynamical model atmospheres. We also discuss the influence of the fundamental model parameters on the resulting synthetic spectra.
\end{abstract}

\section{Introduction}

ISO-SWS spectra of AGB stars allow to study both the molecular features and the dust emission of these stars. Since also most of the AGB stars are (strongly) variable, ISO-SWS data provide important constraints for hydrostatic and dynamic model atmospheres. Carbon stars are of special importance in this respect because the molecular features probably affected by dynamical phenomena fall in the ISO-SWS range and also the theory of dust formation is much better understood than in the O-rich case.

\section{Model spectra}

Synthetic spectra for carbon-rich AGB stars in the wavelength region between 0.5 and $12 \mu \mathrm{m}$ were computed using the improved dynamical model atmospheres of Höfner et al. (1998; also this volume) and using the opacity sampling technique. The molecules $\mathrm{CO}, \mathrm{CH}, \mathrm{C}_{2}, \mathrm{CN}, \mathrm{C}_{3}, \mathrm{HCN}$ and $\mathrm{C}_{2} \mathrm{H}_{2}$ were included. The models are characterized by the following set of parameters: stellar mass,

\footnotetext{
${ }^{1}$ also: Institut für Astronomie, Univ. Vienna
} 
luminosity, effective temperature of the hydrostatic initial model and the carbonto-oxygen abundance $(\mathrm{C} / \mathrm{O})$ ratio as well as the piston period $\mathrm{P}$ and velocity amplitude $\Delta u_{P}$. All abundances except that of carbon are assumed to be solar. Our 'standard' model has the following parameters: $\mathrm{M}=1 \mathrm{M}_{\odot}, \mathrm{L}=7000 \mathrm{~L}_{\odot}$, $\mathrm{T}_{\text {eff }}=2880 \mathrm{~K}, \mathrm{C} / \mathrm{O}=1.4, \mathrm{P}=390 \mathrm{~d}$ and $\Delta \mathrm{u}_{\mathrm{P}}=4 \mathrm{~km} \mathrm{~s}^{-1}$. Table 1 summarizes how the different model parameters change the spectra. A detailed discussion can be found in Loidl et al. (1998).

Table 1. Influence of different model parameters on spectra

\begin{tabular}{l|l|l}
\hline parameter & features & variation \\
\hline T higher & weaker & stronger \\
L higher & stronger & smaller \\
M smaller & stronger & smaller \\
C/O higher & stronger & stronger \\
$\Delta$ up higher $_{\mathrm{P}}$ stronger & stronger \\
no dust & stronger & \\
\hline
\end{tabular}

In Fig. 1 gas pressure versus temperature is plotted for the maximum (full) and the minimum (dashed) phase of our 'standard' dynamical model. The horizontal bars indicate the temperature ranges for the molecules $\mathrm{CO}, \mathrm{C}_{2}, \mathrm{CN}$, $\mathrm{HCN}, \mathrm{C}_{3}$ and $\mathrm{C}_{2} \mathrm{H}_{2}$ where their features between 0.5 and $12 \mu \mathrm{m}$ are approximately formed. The grey shaded area denotes the region of dust formation.

\section{Observational data}

Within our open time observing programs the spectral variations of a few representative AGB stars were monitored with the ISO-SWS (de Graauw et al. 1996). In the following table we list the major characteristics of our targets:

Table 2. Summary of targets

\begin{tabular}{l|l|l|c|c|l}
\hline Name & Type & Spectrum & $\mathrm{P}[\mathrm{d}]$ & $\begin{array}{c}\mathrm{dM} / \mathrm{dt} \\
{\left[10^{-6}\right]}\end{array}$ & Comment \\
\hline T Dra & $\mathrm{M}$ & $\mathrm{C} 6,2-\mathrm{C} 8,3$ & 422 & 2 & typical C-Mira \\
V Cyg & $\mathrm{M}$ & $\mathrm{C} 5,3-\mathrm{C} 7,4$ & 422 & 2 & typical C-Mira \\
V CrB & M & $\mathrm{C} 6,2$ & 358 & 0.2 & metal poor? C-Mira \\
R Scl & SRb & $\mathrm{C} 6,5$ & 370 & $<1$ & large amplit., detached shell \\
TT Cyg & SRb & $\mathrm{C} 5,4 \mathrm{e}$ & 118 & 0.01 & small amplit., detached shell \\
V460 Cyg & SRb & $\mathrm{C} 6,4$ & 180 & 0.1 & small amplit. \\
\hline
\end{tabular}

For the first five targets we have typically five observations distributed over the pulsational cycle. The spectra were full grating scans $(2.4 \mu \mathrm{m}$ to $44 \mu \mathrm{m})$ with a typical resolution of 400 . The pipeline processed data (mostly OLP versions 


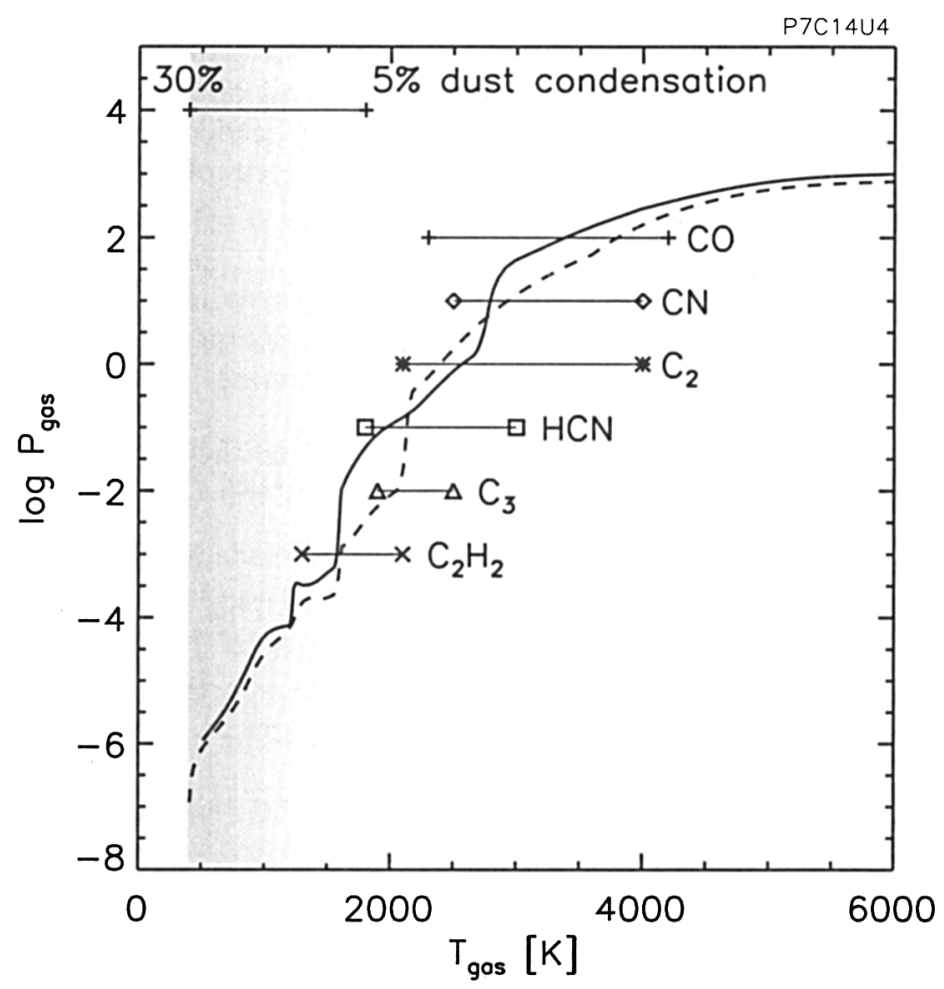

Figure 1. Gas pressure versus temperature for maximum (full) and minimum (dashed) phase of the 'standard' dynamical model. The hrizontal bars indicate the temperature ranges for the molecules $\mathrm{CO}, \mathrm{C}_{2}$, $\mathrm{CN}, \mathrm{HCN}, \mathrm{C}_{3}$ and $\mathrm{C}_{2} \mathrm{H}_{2}$ where their features between 0.5 and $12 \mu \mathrm{m}$ are approximately formed. The grey shaded area denotes the region of dust formation.

6.xx) were further reduced with ISAP and then rebinned to a resolution of 100 . For all spectra a significant offset between the two scan directions was found in band $2 \mathrm{C}(7 \mu \mathrm{m}$ to $12 \mu \mathrm{m})$ which was corrected by rejecting the scan direction with the higher flux. The agreement between spectra of the same star taken within a few days was generally better than $10 \%$. There was also good agreement with (quasi-simultaneous) ground based and IRAS photometry.

First results on $\mathrm{R}$ Scl have been presented by Hron et al. (1998) and will serve as a reference for the other targets.

\section{4. (Almost) Dust-free carbon stars}

The spectra of TT Cyg and V460 Cyg have been compared with R Scl and hydrostatic model atmospheres. For the comparison with the models, the ISO 
data were normalized by linear interpolation between the fluxes at several selected 'pseudo-continuum' points and then scaled to a continuum level of 0.8 to 0.9 . The continuum points were selected on the basis of the synthetic spectra. This normalization is somewhat problematic shortward of $2.8 \mu \mathrm{m}$ due to the lack of data points at shorter wavelengths. The results of the comparison can be summarized as follows:

- The depths of the 3 and $3.8 \mu \mathrm{m}\left(\mathrm{C}_{2} \mathrm{H}_{2}\right)$ features relative to the $5.1 \mu \mathrm{m}$ $\left(\mathrm{C}_{3}\right)$ feature suggest a sequence of decreasing temperature: V460 Cyg $\rightarrow$ $\mathrm{TT} \mathrm{Cyg} \rightarrow \mathrm{R} \mathrm{Scl}$ (maximum) $\rightarrow \mathrm{R} \mathrm{Scl}$ (minimum).

- The ratio of the strength of the $4.6 \mu \mathrm{m}(\mathrm{CO})$ to the $5.1 \mu \mathrm{m}\left(\mathrm{C}_{3}\right)$ features indicates a sequence of increasing $\mathrm{C} / \mathrm{O}$ from V460 Cyg $(1.1<\mathrm{C} / \mathrm{O} \leq 1.4)$ to $\mathrm{TT} \mathrm{Cyg}$ and $\mathrm{R} \mathrm{Scl}$ (both with $\mathrm{C} / \mathrm{O} \approx 1.4$ ).

- The best fits are obtained for $\mathrm{T}_{\text {eff }}=3200 \mathrm{~K}$. This is in agreement with results from the Infrared Flux Method (Ohnaka \& Tsuji 1996) but significantly hotter than what is expected from the spectral type/temperature relation derived from angular diameters (Dyck et al. 1996).

- Even for $\mathrm{T}_{\text {eff }}=3200 \mathrm{~K}$ the hydrostatic models give too strong features at 3 and $3.8 \mu \mathrm{m}$ and a too weak $4.6 \mu \mathrm{m}$ feature.

- The region from 6 to $10 \mu \mathrm{m}$ is generally not well reproduced.

The discrepant feature strengths shortward of $6 \mu \mathrm{m}$ could be due to opacity problems or a non-hydrostatic $\mathrm{T} / \mathrm{P}$-structure (as e.g. caused by shock waves). At longer wavelengths, instrumental problems, the contribution from dust and opacity problems are possible causes. We note that contrary to Aoki et al. (1998), our models always predict a significant (50\%) $\mathrm{C}_{2} \mathrm{H}_{2}$ contribution to the $3 \mu \mathrm{m}$ feature.

\section{Dusty carbon stars}

For the comparison with the dynamic models, a correction of the observed spectra for the dust emission is of course necessary. For this purpose we have used the publicly available DUSTY code (Ivezić et al. 1997). Using selected synthetic spectra for the photospheric input radiation, a ratio of amorphous carbon to $\mathrm{SiC}$ of $4: 1$ and a $1 / \mathrm{r}^{2}$ dust density law, we fitted the full SWS spectra at selected phases. The best fits were obtained with a condensation temperature of $1000 \mathrm{~K}$ and optical depths at $2.2 \mu \mathrm{m}$ between 0.1 (maximum phases) and 0.3 to 0.5 (minimum phases). Since the amount of dust emission for a given ISO spectrum did not really depend on the input spectrum (as long as a realistic synthetic spectrum was used), we constructed smooth dust continua for several optical depths. The typical contribution of these continua to the total flux ranged from $40 \%$ at $4 \mu \mathrm{m}$ to $90 \%$ at $10 \mu \mathrm{m}$. The dust continua were then subtracted from the observed spectra and these spectra were then normalized in the same fashion as described above. We note that due to this procedure, the $\mathrm{SiC}$ feature is not 
subtracted, only the $\mathrm{SiC}$ continuum. Furthermore, the use of a synthetic spectrum assumes that all the molecular features form inside the dust condensation radius, an assumption which seems to be justified on the basis of Fig. 1.

In Fig. 2 we show corrected and normalized spectra of T Dra for two phases together (thick lines) with synthetic spectra based on dynamic model atmospheres. The results from comparing our spectra with several models can be summarized as follows:

- There seems to be a sequence of decreasing $\mathrm{T}_{\mathrm{Max}}$ : TT Cyg $\rightarrow \mathrm{V} \mathrm{CrB} \rightarrow$ T Dra \& V Cyg $\rightarrow$ R Scl.

- The weaker $5.1 \mu \mathrm{m}$ feature in the Miras indicates a sequence of increasing C/O: V CrB \& V460 Cyg $\rightarrow$ V Cyg \& T Dra $\rightarrow$ TT Cyg \& R Scl.

- The three Miras show similar variability patterns: the deepest features appear near visual phase 0.6 , the amplitudes increase from the $5.1 \mu \mathrm{m}$ to the $3 \mu \mathrm{m}$ and the $3.8 \mu \mathrm{m}$ features.

- The agreement with the dynamical models is comparable to what was found for R Scl. A model with $7000 L_{\odot}$ fits better than one with $10000 L_{\odot}$.

- The $14 \mu \mathrm{m}$ feature is comparable to the $3 \mu \mathrm{m}$ feature in depth and variation, which is in agreement with preliminary models for this feature.

- The SiC feature might have a slightly lower contrast near minimum light. Its shape differs systematically from the DUSTY models. This effect is most likely due to the influence of molecular features which means that the interpretation of the $10 \mu \mathrm{m}$ feature requires a proper treatment of the molecules and the dust!

Acknowledgments. This work is supported by the Austrian Science Fund (FWF projects S7308 and J1487), the Danish Natural Science Research Council and the Austrian Academy of Sciences (APART programme). It is a pleasure to thank the ISO operations and instrument teams for their support.

\section{References}

Aoki W., Tsuji T., Ohnaka K., 1998, A\&A 340, 222

de Graauw T., Haser L.N., Beintema D.A., et al., 1996, A\&A 315, L49

Dyck H.M., van Belle G.T., Benson J.A., 1996, AJ 112, 294

Ivezić Ż., Nenkova M., Elitzur M., 1997, User Manual for DUSTY,

Internal Report, University of Kentucky, accessible at http://www.pa.uky.edu/ moshe/dusty

Hron J., Loidl R., Höfner S., et al., 1998, A\&A 335, L69

Loidl R., Höfner S., Jørgensen U.G., Aringer B., 1998, A\&A, submitted

Ohnaka K., Tsuji T., 1996, A\&A 310, 933 


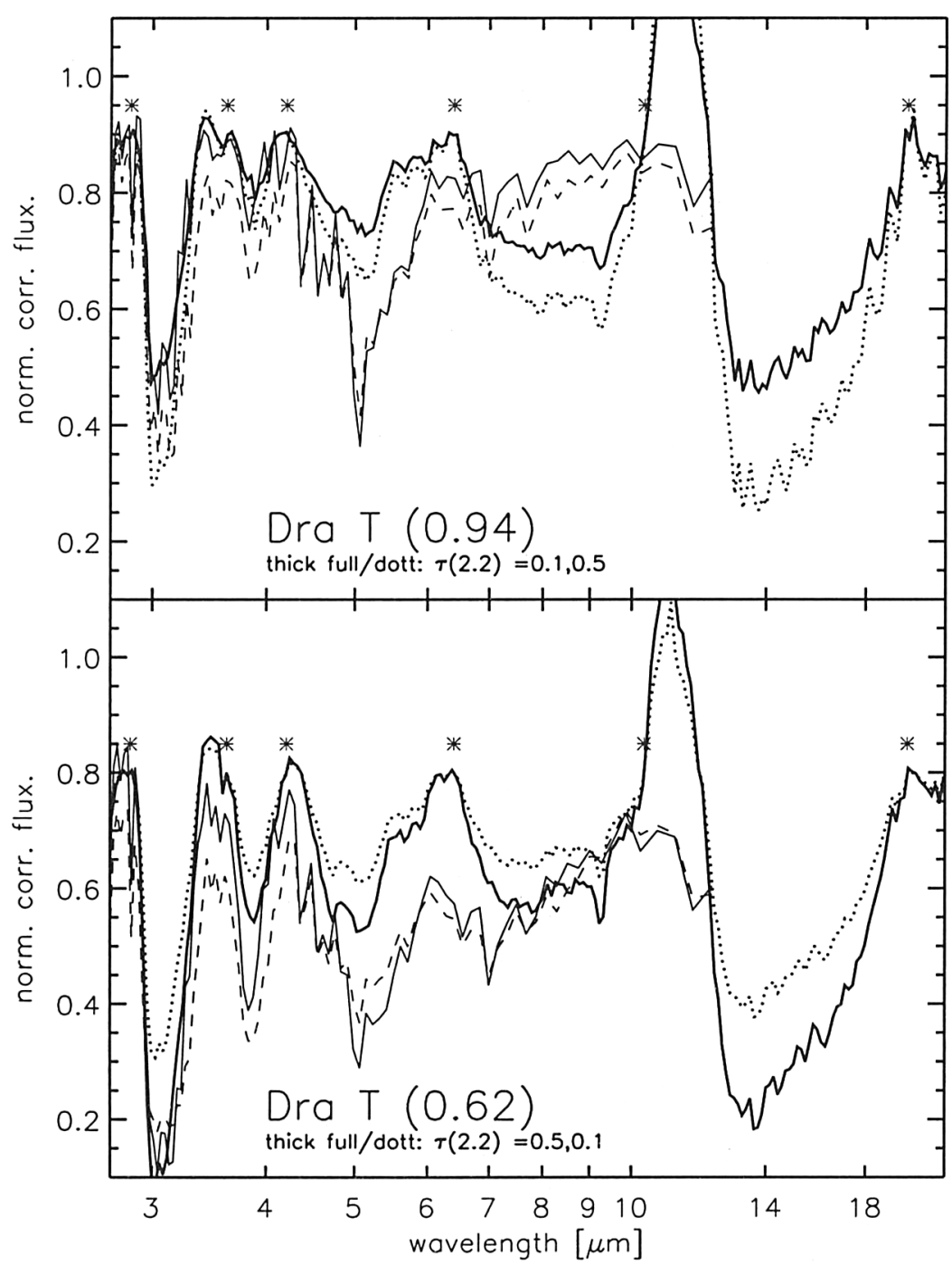

Figure 2. ISO-SWS spectra of T Dra taken near visual phases 0.94 (top) and 0.62 (bottom) compared to synthetic spectra. The observed spectra were normalized and corrected for continuous dust emission (Sect. 5) and are shown with thick full and dotted lines. The dust optical depths at $2.2 \mu \mathrm{m}$ are indicated in the plot, the thick full lines result from the best DUSTY fit, the thick dotted lines give an indication for the influence of this parameter on the depth of the molecular features. The thin full lines are the synthetic spectra at similar phases for the 'standard' dynamical model described in the text, the thin dashed lines are for a model with $10000 \mathrm{~L}_{\odot}$. The asterisks mark the wavelengths used for the normalization. 